\title{
Relación docente-estudiante y compromiso escolar: percepción de estudiantes secunda- rios con necesidades educativas especiales
}

\author{
Gabriela Lara ${ }^{\mathrm{a}}$ \\ Universidad de Concepción, Chile \\ Nicole González ${ }^{b}$ \\ Universidad de Concepción, Chile \\ Francisca Lara ${ }^{\mathrm{C}}$ \\ Universidad de Concepción, Chile
}

\author{
Lorena Lagos ${ }^{d}$ \\ Universidad de Concepción, Chile \\ Victoria Parra, Ph. D. ${ }^{\mathrm{e}}$ \\ Universidad Católica de Temuco, Chile \\ Claudia Paz Pérez-Salas, Ph. D. ${ }^{\dagger}$ \\ Universidad de Concepción, Chile
}

cperezs@udec.cl

\section{Resumen (analítico)}

Investigaciones previas sostienen que la relación docente-estudiante es un factor relevante para el compromiso escolar y rendimiento académico. Sin embargo, su impacto ha sido poco explorado en estudiantes con necesidades educativas especiales. Este estudio examina la influencia de la relación docente-estudiante en el compromiso escolar según la percepción de estudiantes de primer año medio con necesidades educativas especiales de una Región de Chile. Se realizó un estudio cualitativo, de tipo fenomenológico y de alcance descriptivoanalítico. Se desarrollaron diez entrevistas en profundidad a estudiantes con necesidades educativas especiales, las que se analizaron mediante el método de comparación constante de la teoría fundamentada. Los resultados revelan el rol clave de los/as docentes en el compromiso escolar a través de la satisfacción de las necesidades de vinculación y competencia de sus estudiantes, pero no de su autonomía.

\section{Palabras clave}

Compromiso escolar, necesidades educativas especiales, relación profesor-estudiante, educación secundaria.

\section{Thesauro}

The thesaurus for education systems in Europe.

\section{Para citar este artículo}

Lara, G., González, N., Lara, F., Lagos, L., Parra, V., \& Pérez-Salas, C. P. (2022). Relación docente-estudiante y compromiso escolar: percepción de estudiantes secundarios con necesidades educativas especiales. Revista Latinoamericana de Ciencias Sociales, Niñez y Juventud, 20(1), 1-24.

https://dx.doi.org/10.11600/rlcsnj.20.1.4495

\section{Historial}

Recibido: 05.03.2021

Aceptado: 05.08.2021

Publicado: 02.12.2021

\section{Información artículo}

Este artículo contó con financiamiento de la Agencia Nacional de Investigación y Desarrollo de Chile. [Anid/Conicyt, Proyecto Fondecyt Regular 1181265]. Investigadora Responsable Claudia P. Pérez-Salas. Área: psicología. Subárea: psicología educacional. 


\section{Teacher-student relationship and student engagement: perceptions of secondary students with special educational needs}

Abstract (analytical)

The results of existing research studies show that the teacher-student relationship is a relevant factor that affects academic engagement and school performance. However, there is limited evidence of its impact on students with special educational needs. This study examines the influence of the teacherstudent relationship on school engagement based on the perceptions of students with special educational needs in their first year of high school education in Chile. The study used a qualitative, phenomenological, and descriptive-analytical design. A total of ten in-depth interviews were conducted with students with special educational needs. The data was analysed using the methodological guidelines of grounded theory. The results highlight the key role of teachers in academic engagement by satisfying students' psychological needs in the areas of connection and competence, but not satisfying students' need for autonomy.

Keywords

Learner engagement, special needs students, teacher-student relationship, secondary school students.

\section{Relação professor-aluno e compromisso escolar: percepção de jovens com necessidades educacionais especiais}

Resumo (analítico)

Pesquisas anteriores afirmam que a relação professor-aluno é um fator relevante para o engajamento e desempenho acadêmico. No entanto, seu impacto tem sido pouco explorado em alunos com necessidades educacionais especiais. Este estudo examina a influência da relação professor-aluno no compromisso escolar segundo a percepção dos alunos com alunos com necessidades educacionais especiais do primeiro ano do ensino médio, da Chile. O desenho foi qualitativo, fenomenológico, descritivo-analítico em escopo. Foram realizadas 10 entrevistas em profundidade com alunos com necessidades educacionais especiais. Os dados foram analisados segundo as diretrizes metodológicas da teoria fundamentada nos dados. Os resultados apontam para o papel fundamental do professor no comprometimento escolar, por meio da satisfação das necessidades de vinculação e competência de seus alunos, mas não de sua autonomia.

\section{Palavras-chave}

Compromisso escolar, necessidades educacionais especiais, relação professor-aluno, estudantes do ensino médio.

\section{Información autoras}

[a] Psicóloga, Universidad de Concepción. (iD) 0000-0002-3595-6542. H5: 0. Correo electrónico: gabrlara@udec.cl [b] Psicóloga, Universidad de Concepción. iD 0000-0002-2043-0943. H5: 0. Correo electrónico: nicolgonzalezr@udec.cl

[c] Psicóloga, Universidad de Concepción. (iD) 0000-0002-5201-8072. H5: 0. Correo electrónico: flara@udec.cl

[d] Psicóloga, Universidad de Concepción. iD 0000-0002-8808-9665. H5: 0. Correo electrónico: lorenalagos@udec.cl

[e] Psicóloga. Doctora en Educación, Universidad de Columbia (Estados Unidos). Profesora Departamento de infancia y educación básica, Universidad Católica de Temuco, Chile. iD 0000-0001-8870-2629. H5: 3. Correo electrónico: vparra@uct.cl

[f] Psicóloga. Doctora en Psicología, Pontificia Universidad Católica de Chile. Profesora Departamento de psicología, Universidad de Concepción, Chile. iD 0000-0002-6940-6514. H5: 8. Correo electrónico: cperezs@udec.cl 


\section{Introducción}

urante sus trayectorias educativas, los y las estudiantes enfrentan múltiples
desafíos de adaptación y negociación de conductas académicas y sociales. El éxito o fracaso en el abordaje de tales demandas influye tanto en el aprendizaje como en el ajuste psicológico (Li \& Learner, 2011; Wang \& Fredericks, 2014). La evidencia indica que estudiantes con necesidades educativas especiales (en adelante NEE) presentan dificultades para enfrentar dichos desafíos, generando en algunos casos un impacto negativo en los resultados académicos, disciplina escolar y bienestar subjetivo (Cortiella \& Horowitz, 2014; Douglas et al., 2012; Moreira et al., 2015; Schindler, 2018).

La escuela es una institución social importante donde los adolescentes ponen a prueba sus habilidades sociales y van desarrollando su propia identidad (Erentaite et al., 2018; Nakkura, 2020). En este contexto, resulta relevante examinar la influencia del vínculo docente-estudiante sobre el compromiso escolar y el rendimiento académico, relación que ha sido constatada en investigaciones previas (e. g. Quin, 2017; Roorda et al., 2011). No obstante, gran parte de los estudios se han enfocado en conocer a profundidad esta relación en estudiantes con desarrollo típico (Ewe, 2019; O’Donnell \& Reschly, 2020) y los escasos estudios que han analizado el efecto de esta relación en estudiantes con NEE no han mostrado resultados concluyentes (O’Donnell \& Reschly, 2020; Roorda et al., 2011).

Según Wang et al. (2019), el compromiso escolar es un constructo que posee cuatro dimensiones: a) la dimensión conductual, que se manifiesta en una conducta apropiada, productividad y participación en el trabajo académico y extra-académico; b) la dimensión emocional, que considera las reacciones afectivas positivas hacia la escuela, incluyendo el disfrute y valor atribuido al trabajo escolar; c) la dimensión cognitiva, que incluye la reflexión, el uso de estrategias de aprendizaje profundo y la disposición a esforzarse por comprender ideas complejas; y, d) la dimensión social, que se refiere a la calidad de las interacciones sociales con los compañeros tanto en el contexto de la sala de clases (Rimm-Kaufman et al., 2016; Wang et al., 2019) como fuera de ella (Wang et al., 2019). 
La presencia de reacciones y procesos desadaptativos en cada una de estas dimensiones da cuenta de un fenómeno denominado «desconexión escolar» (disengagement) (Skinner et al., 2009; Wang et al., 2019).

El compromiso escolar está determinado por variables individuales y el contexto de los y las estudiantes (Christenson et al., 2012). Entre los factores personales que influyen en el desarrollo del compromiso se han identificado el sexo y la edad, demostrándose que estudiantes hombres poseen un grado de compromiso escolar más bajo que estudiantes mujeres (Wang \& Eccles, 2012; Wang et al., 2011); y que quienes se encuentran finalizando la educación secundaria muestran un nivel de compromiso escolar menor en comparación con sus pares que se encuentran cursando los primeros años, constatándose que el compromiso escolar decrece durante la adolescencia (Burns et al., 2018; Engels et al., 2021).

En conclusión, las variables individuales (v. g. sexo y edad) y contextuales (v. g. relación con los pares, padres y docentes) influyen en el compromiso escolar (Wang \& Eccles, 2013) y la interacción entre estos factores puede influir en las reacciones positivas y negativas que el o la estudiante desarrollará hacia el contexto escolar como, por ejemplo, interés, aburrimiento y ansiedad (Fredricks, 2015; Moreira et al., 2015; Ramos-Díaz et al., 2016).

De acuerdo con el modelo del sistema del sí-mismo (Connell \& Wellborn, 1991; Ryan \& Deci, 2017; Skinner \& Pitzer, 2012), los factores individuales y contextuales influyen en las distintas variantes de compromiso y desconexión de los y las estudiantes, a partir de la manera en que el contexto escolar ayuda a satisfacer tres necesidades relevantes para el individuo: las necesidades de vinculación, de autonomía y de competencia. La necesidad de vinculación se refiere a la manera en la que el individuo se siente seguro, conectado y valorado por otros; la necesidad de autonomía se relaciona con la necesidad de experimentar agencia sobre el propio comportamiento, tanto en su iniciación y regulación como en el mantenimiento de la actividad; y la necesidad de competencia se relaciona al grado en que el individuo sabe cómo obtener ciertos resultados positivos y evitar los negativos. Así, el compromiso escolar se conceptualiza como parte de un proceso motivacional más amplio, donde el contexto de aprendizaje alimenta la conceptualización que el individuo tiene respecto a sí mismo (Fredricks et al., 2019). Cuando las necesidades psicológicas son satisfechas en el contexto escolar el compromiso emerge, lo que se manifiesta en la emoción, conducta y cognición. Por el contrario, si las necesidades psicológicas no son satisfechas surge la desconexión en el individuo (Connell \& Wellborn, 1991).

Como se señaló anteriormente, una de las variables contextuales que influye en el compromiso escolar es la relación docente-estudiante (Engels et al., 2021; Pérez-Salas et al., 
2021; Quin, 2017; Reeve et al., 2004; Roorda et al., 2011; Wang et al., 2013). El impacto de la relación docente-estudiante en jóvenes con NEE ha sido analizada desde la hipótesis del riesgo académico (Hamre \& Pianta, 2001), a partir de la cual se concluye que los y las estudiantes con bajo nivel socioeconómico, pertenecientes a minorías o con dificultades de aprendizaje, se verán más afectados en su compromiso escolar por la calidad de la relación que establezcan con sus docentes. Un meta-análisis realizado por Roorda et al. (2011) encontró soporte a esta hipótesis y reportó que el efecto de una mala relación docente-estudiante era más perjudicial para el compromiso escolar y logro académico de estudiantes en riesgo de fracasar que para quienes no tenían esta condición.

En el caso de jóvenes con NEE, el efecto de la relación docente-estudiante podría explicarse a partir de los hallazgos de otros estudios, los cuales sostienen que las actitudes docentes promueven un mayor compromiso en el aula. Ejemplo de estas actitudes son la cercanía física, la atención individualizada, la interacción uno a uno durante la clase (Blatchford et al., 2009), la comprensión de la condición del estudiante con NEE (de Verdier et al., 2017) y el apoyo a la autonomía del estudiante (Alley, 2019; Reeve et al., 2004). No obstante lo anterior, Blatchford et al. (2009) y Carter et al. (2008) concuerdan en señalar que una mayor proximidad docente-estudiante puede afectar la socialización con los pares, dado que el o la estudiante con NEE se vinculará durante gran parte de la jornada principalmente con sus docentes en desmedro de la relación con sus pares.

Con respecto a los y las docentes, se ha reportado que ciertas conductas y actitudes docentes como, por ejemplo, el uso de un nivel moderado de dirección, sensibilidad y una actitud receptiva, se asocian a mayores niveles de compromiso escolar en estudiantes con NEE (Ben-Yehuda et al., 2009; Kiefer et al., 2015; Rogers et al., 2015). Además, la relación y proximidad que establezca el/la docente con jóvenes con NEE depende de su actitud hacia la inclusión y experiencias previas con otros estudiantes con NEE (BenYehuda et al., 2009; Forlin \& Chambers, 2011).

En síntesis, la evidencia permite concluir que las prácticas y el estilo personal del docente pueden influir en el compromiso escolar en jóvenes con NEE (Blatchford et al., 2009; Moreira et al., 2013; de Verdier et al., 2017). Pese a ello, parece no existir claridad sobre el impacto específico de la relación docente-estudiante en estudiantes con NEE (Roorda et al., 2011), ni tampoco haber sido abordado desde la perspectiva de los propios estudiantes, aspecto que se pretendió indagar en esta investigación.

El presente estudio se sustenta en el paradigma constructivista con un enfoque cualitativo de tipo fenomenológico (Hernández-Sampieri \& Mendoza, 2018). El propósito 
de la investigación fue analizar la influencia de la relación docente-estudiante en el compromiso escolar en adolescentes de educación secundaria con NEE desde la perspectiva de los y las estudiantes. Para ello, se realizaron entrevistas orientadas por la siguiente pregunta de investigación: ¿cómo influye la relación docente-estudiante en el compromiso escolar de estudiantes con NEE? Se espera que los resultados de este trabajo brinden información útil a investigadores, docentes y profesionales que trabajan con estudiantes con NEE.

\section{Método}

\section{Diseño}

El presente estudio cualitativo se inserta dentro de una investigación de diseño cuantitativo, formando parte de una investigación mayor con un diseño anidado concurrente de un modelo dominante (Creswell, 2011). El estudio que se reporta en este artículo permitió profundizar en el conocimiento de las variables que forman parte del estudio principal. Mediante la implementación de una metodología descriptiva analítica de carácter cualitativo (Flick, 2004, Maxwell, 2019), se profundizó desde una perspectiva fenomenológica en las perspectivas y significados que los y las estudiantes con NEE confieren a sus experiencias escolares (Hernández-Sampieri \& Mendoza, 2018).

\section{Participantes}

Diez estudiantes de primer año medio (ocho mujeres y dos hombres) participaron voluntariamente en este estudio y constituyen una submuestra del estudio mayor de tipo cuantitativo descrito previamente. Los y las participantes debían cumplir con los siguientes criterios de inclusión y exclusión: a) ser estudiantes de enseñanza media pertenecientes a escuelas regulares municipales de la región del Biobío y b) pertenecer al Programa de Integración Escolar de su establecimiento debido a NEE de funcionamiento intelectual limítrofe o dificultades específicas del aprendizaje, de acuerdo a los criterios de diagnóstico exigidos por el Ministerio de Educación de Chile. El criterio de exclusión fue la presencia de trastorno del espectro autista concomitante, dado que una de las características de este tipo de NEE es la dificultad de socialización, lo que podría estar relacionado con el compromiso escolar. 


\section{Técnicas y procedimientos de recolección de datos}

Se recurrió a la entrevista en profundidad como un instrumento de recolección de datos, dado que posibilita una comunicación directa entre entrevistador y entrevistado, estableciendo una relación que permite aproximarse a las ideas y sentimientos de los y las participantes, incluyendo sus motivaciones, deseos, creencias y esquemas de interpretación, los cuales se actualizan durante la entrevista (Canales, 2006).

Se diseñó una entrevista semiestructurada para generar instancias de diálogo con los y las participantes del estudio, permitiendo la aclaración de términos específicos, la identificación de ambigüedades y la reducción de formalismos (Díaz-Bravo et al., 2013; Maxwell, 2019). A su vez, esta herramienta permite tomar decisiones durante el desarrollo de la entrevista que respondan su curso y a las necesidades de profundizar en algunos aspectos clave. El guión de la entrevista fue desarrollado empleando una matriz de coherencia que se definió en función de la relación entre las preguntas de la entrevista y los objetivos de investigación. Se realizó una entrevista piloto que permitió ajustar la cantidad de preguntas y su redacción.

La entrevista se organizó en cuatro apartados. En el primero se identificó y caracterizó al entrevistado; en el segundo, se indagó en torno a las experiencias y participación del estudiante en actividades escolares; el tercer apartado se refería a las opiniones sobre la relación docente-estudiante; $\mathrm{y}$, finalmente, el cuarto examinó la percepción de los y las estudiantes sobre prácticas pedagógicas de sus docentes.

Las entrevistas se realizaron de manera individual en un espacio facilitado para ello en los establecimientos educacionales a los que asistían los y las participantes. Dos investigadoras realizaron las entrevistas, las cuales se registraron en audio y fueron posteriormente transcritas para su análisis. La duración de las entrevistas osciló entre los zo y 40 minutos. Todos los apoderados entregaron un consentimiento informado para autorizar la participación de los y las estudiantes y estos/as dieron su asentimiento para participar en el estudio que se reporta en este artículo.

\section{Procedimiento de análisis de información}

El análisis de datos fue realizado siguiendo los lineamientos metodológicos de la teoría fundamentada (Strauss \& Corbin, 1994), la cual, mediante un procedimiento sistemático de comparación constante, se orienta a conformar una teoría que permita responder a las preguntas de investigación. 
Los datos obtenidos a través de las entrevistas fueron analizados utilizando el software de análisis Dedoose, utilizando los procedimientos metodológicos propuestos por Glaser y Strauss (1967) en dos etapas: una primera, de codificación abierta, mediante lectura profunda de las transcripciones de las entrevistas realizadas a los/as participantes y posterior clasificación en categorías, permitió identificar conceptos vinculados con la relación docente-estudiante. La segunda, de codificación axial, consistió en establecer relaciones entre las categorías previamente definidas. Se realizó la triangulación de datos entre las investigadoras en ambas etapas.

\section{Resultados}

Los códigos emergentes del análisis fueron organizados en dos categorías comprensivas alineadas a los objetivos de investigación y al marco teórico: condicionantes de la relación docente-estudiante y compromiso escolar.

\section{Condicionantes de la relación docente-estudiante según la percepción de los y las estudiantes}

Los y las estudiantes con NEE describen la relación con sus docentes de manera diversa, estableciendo relaciones positivas o negativas con ellos y ellas. De acuerdo con los hallazgos, la naturaleza de esta relación se vincula a tres dimensiones: a) características de las prácticas profesionales del docente; b) características personales del o la docente; y c) el rol que ejerce el o la docente en el establecimiento según la política de inclusión que rige el programa de integración.

\section{Características de las prácticas profesionales de los y las docentes}

Según la percepción de los y las entrevistados, algunas prácticas de sus docentes como, por ejemplo, la metodología que emplean para desarrollar las clases y el tipo de interacción que promueven en ellas, inciden en la relación que desarrollan con sus profesores/as. En primer lugar, los y las entrevistadas señalan tener una mejor relación con docentes que explican bien los contenidos; ello se evidencia, por ejemplo, en situaciones en las cuales los y las docentes dan respuestas adecuadas a las preguntas de sus estudiantes hasta resolverlas a cabalidad. Además, destacan positivamente la práctica de hacer clases didácticas y utilizar recursos innovadores, evitando las clases expositivas. Según los y las estudiantes, cuando sus docentes realizan acciones como las anteriores, sienten 
mayor motivación, ven incrementada su atención y participan con mayor frecuencia durante las clases. Esto se puede observar en lo señalado por un estudiante:

Enseñan bien, algunos (...); son didácticos, así como para explicar las clases y todo eso... Eh, no sé porque tienen una paciencia para algunos, así como que le explican como una, dos, tres veces así. (Estudiante 3, hombre, 16 años)

El fragmento anterior evidencia, a su vez, que las prácticas docentes tienen el potencial de generar, no solo un ambiente de trabajo, sino un clima emocional de acogida a las necesidades de los estudiantes, tema que se abordará en los apartados siguientes.

Por otra parte, los y las estudiantes señalan que la didáctica de la clase y la comunicación con sus docentes puede impactar negativamente la relación docente-estudiante. En el primer caso, señalan tener dificultades para establecer una buena relación con docentes que hacen clases monótonas y poco motivadoras. En cuanto a la comunicación, manifiestan tener dificultades para relacionarse con docentes que no permiten una comunicación efectiva, lo que se manifiesta en no responder a sus consultas o no brindar respuestas satisfactorias. Un estudiante ejemplifica lo anterior:

Me acuerdo que una vez di la opinión con la profe de Química y me dijo que no, que no. Cómo no iba a saber eso si es algo básico y al final como que me terminó retando; entonces para qué voy a dar la opinión en su materia. Entonces mejor me ahorro el comentario. (Estudiante 6, mujer, 15 años)

\section{Características personales de los y las docentes}

Los y las estudiantes identifican características personales de los y las docentes que generan relaciones positivas entre ellos. Mencionan con frecuencia características como la amabilidad, la capacidad para inspirar confianza (especialmente al momento de tratar temas personales) y el permitir el espacio al humor y a la distensión en sus clases. Las características destacadas por los estudiantes dan cuenta de conductas de los docentes que permiten establecer vínculos más allá de lo académico. Una estudiante comenta:

La profe $\mathrm{C}$, es que con ella como que hay mucha confianza; es como segunda mamá porque, como les dije, no se preocupa solamente de nuestras notas, sino por nuestro bienestar y es como súper buena onda; es como alegre y, como es joven, no es como tan amargá. Entonces es como muy bacán (...); a veces dan ganas como de venir pa'cá solamente pa poder hablar con la profe $\mathrm{C}$, porque a veces antes de que toquen da como 5 minutos, entonces ahí 
se pone a hablar con nosotras en buena onda; pregunta cómo estoy. Entonces como que igual me dan ganas de venir como por ella. (Estudiante 6, mujer, 15 años)

En el otro extremo, los estudiantes señalan tener dificultades para establecer una buena relación con docentes que son percibidos como poco simpáticos, con falta de paciencia y quienes usan un tono de voz demasiado fuerte o autoritario. «Algunos explican con toda la amabilidad del mundo, pero otros no (...); como que se enojan eh, al explicarlo como, más de una vez» (Estudiante 7 , mujer, 15 años).

En menor medida los y las estudiantes hacen referencia a conductas de desorganización de los y las docentes (por ejemplo, perder trabajos de los estudiantes), lo que afecta negativamente la percepción que tienen de ellos o ellas.

\section{Rol de el o la docente en el establecimiento educativo}

El análisis da cuenta de diferencias existentes en la relación que los estudiantes tienen con sus docentes, dependiendo de si son docentes de aula o docentes del Programa de Integración Escolar (PIE). En el caso de los primeros, los y las estudiantes describen relaciones tanto positivas como negativas, que responden a las características de las prácticas profesionales y las características personales descritas en las dimensiones anteriores.

En el caso particular de los y las docentes PIE, si bien los y las estudiantes igualmente explican la naturaleza de la relación a partir de las características anteriores, el contexto en el cual se dan dichas interacciones parece tener un rol mediador relevante. De forma generalizada, los estudiantes destacan una buena relación con los docentes PIE, lo cual atribuyen a la interacción personalizada que se da en el marco del programa. En este contexto, los entrevistados destacan percibir apoyo de parte de estos docentes y hacen referencia a que les enseñan en un contexto de privacidad, caracterizando la situación como una instancia que les permite una mayor comprensión de los temas relacionados con el ámbito académico. Al respecto, una estudiante señala:

Es que es distinto porque con ella entonces están todos po, y cuando yo vengo con ella como que ella me hace cualquier pregunta y yo como que le respondo así; como que le tengo más confianza, no sé; como que yo a cualquier persona que esté sola con ella me hace preguntas y yo como que le respondo al tiro. (Estudiante 3, mujer, 16 años)

Los elementos anteriores dan cuenta de que los estudiantes, al reflexionar sobre la naturaleza de las relaciones con sus docentes, ponen acento en las conductas y discursos por parte de sus docentes, tanto los vinculados a la dimensión académica como interpersonal. 


\section{Compromiso escolar}

El segundo objetivo de la investigación fue examinar las distintas dimensiones que conforman el compromiso escolar. Los entrevistados describen su compromiso escolar, aludiendo a diversos comportamientos, situaciones, emociones y pensamientos en relación a su establecimiento educativo y al trabajo académico; estos pueden ser agrupados en función de los cuatro factores: compromiso comportamental, compromiso emocional, compromiso cognitivo (Fredricks et al., 2004) y compromiso social (Wang et al., 2019).

\section{Compromiso comportamental}

En esta dimensión se agrupan una serie de comportamientos que los entrevistados vinculan a la participación en actividades académicas. Esta es entendida como el involucramiento y participación que tienen tanto en actividades académicas dentro y fuera del aula en horario regular, así como en actividades extracurriculares impartidas en sus establecimientos educativos. Es importante señalar que los y las estudiantes perciben que el grado de involucramiento y participación varía en función de dos variables: el interés que posean sobre la materia y la relación que mantienen con el o la docente.

El comportamiento mencionado con mayor frecuencia por los entrevistados es la conducta en clase. Una buena conducta (v. g. ayudar a el o la docente a repartir los materiales de la clase o participar en actividades de la clase) se asocia a participación. Un estudiante ejemplifica la participación en clases de esta forma: «Por ejemplo, matemáticas cuando la profesora nos dice, sumen esto, y yo lo hago rápidamente y le digo el resultado» (Estudiante 2, hombre, 15 años).

Por el contrario, una mala conducta en clases involucra poca o nula participación en las actividades. Los y las estudiantes señalan que una mala conducta se explicaría por diversos elementos; entre ellos, una mala relación con el o la docente, no sentir interés en la materia y tener dificultades para comprenderla. Como consecuencia, los estudiantes comentan incurrir en conductas de desconexión, tales como conversar con compañeros o ponerse audífonos para escuchar música.

Otro comportamiento que los estudiantes asocian a una forma de participación es preguntar en clases, solicitando la atención de los docentes para comprender y aclarar contenidos, tanto a nivel individual (atención personalizada al estudiante en su puesto) como a nivel de curso completo. Preguntar en clases se evidencia en comportamientos como levantar la mano para señalar la inquietud o acercarse al docente para aclarar dudas. Algunos entrevistados señalan que en ocasiones evitan preguntar en clases pese a 
tener dudas, debido a diversas razones, siendo la más recurrente el sentir vergüenza o timidez y, en segundo lugar, por considerar que el o la docente no está dispuesto a atender sus dudas.

Entrevistadora: ¿Por qué crees que te has sacado malas notas?

Estudiante: Porque yo no soy de preguntar a los profes cuando yo no entiendo.

Entrevistadora: Cuando tienes una duda, ¿no haces la pregunta al profesor?

Estudiante: No porque me da vergüenza. (Estudiante 7, mujer, 15 años)

Otro aspecto mencionado por los estudiantes es la capacidad de responder en clases como manifestación de la participación/no participación. Los estudiantes mencionan que el responder en clases dependería, entre otras cosas, de si el o la estudiante conoce o no los contenidos de la pregunta, el des/interés del estudiante por la asignatura o sus contenidos, la percepción de que el o la docente no les dé el tiempo suficiente para responder o simplemente no querer entregar opiniones personales en clases.

Las ideas anteriores indican que la dimensión compromiso comportamental de los estudiantes se evidencia en una serie de conductas, y la presencia de dichas conductas se vincula con las características personales de cada estudiante, la relación que perciben tener con sus docentes o el apoyo recibido de parte de estos en el contexto de las actividades escolares.

\section{Compromiso emocional}

El compromiso escolar emocional o afectivo refiere a las reacciones emocionales y el disfrute atribuido al trabajo escolar (Fredricks et al., 2004). Este se configura a partir de las relaciones y actividades que realizan los estudiantes en sus establecimientos educativos (Christenson et al., 2012).

Al respecto, los entrevistados manifiestan tanto reacciones emocionales positivas y negativas asociadas a su asistencia al establecimiento y su participación en actividades escolares. Destaca principalmente la existencia de sentimientos positivos como sentirse apoyados, lo que indican se da en la relación con sus docentes, sus pares y sus familias. Específicamente, en relación a los docentes, los estudiantes destacan sentirse escuchados por algunos de ellos para comentar sus dudas sobre lo visto en clase contando con espacios de comunicación cuando necesitan consejos y ayuda; y que son apoyados tanto en temas personales, familiares y de convivencia escolar. Los estudiantes mencionan diversos ejemplos del apoyo docente: 
Entrevistadora: ¿Cuéntame cómo es esta relación? Dices que es buena, pero ¿por qué es buena?

Estudiante: Porque podemos, no sé, lesear con ellos, y conversar pu, conversar así con confianza; pero no obviamente con groserías y esas cosas, así bien pu, así reírnos con él [docente]. (Estudiante 10, mujer, 15 años)

Entrevistadora: ¿Por qué crees que tienes una relación mejor con ella?

Estudiante: porque, hablamos más (...) de problemas, de la vida, de todo. (Estudiante 1, mujer, 14 años)

Otro de los sentimientos positivos mencionado es sentirse tratados con igualdad en el contexto escolar. Al respecto mencionan que en general los docentes no hacen diferencias entre los estudiantes $y$, por ende, se sienten tratados igual que al resto de sus compañeros y compañeras que no tienen NEE. En cuanto a sus pares, manifiestan no percibir diferencias entre sí mismos y sus compañeros; es decir, se sienten iguales, a pesar de tener NEE y recibir apoyo del equipo PIE.

En contraste, algunos entrevistados mencionan emociones negativas asociadas a sus experiencias educativas. En cuanto a los docentes, los estudiantes comparten no sentirse escuchados en ocasiones, lo que asocian a que algunos docentes no tienen disposición para atenderlos y que les hacen sentir que son una molestia. Al respecto un estudiante describe lo siguiente:

Algunos son pesados. No sirven para ser profesores, porque se supone que uno para ser profesor tiene paciencia, y tiene tiempo pu. Esta señora anda pesá todo el rato y nos están retando. (Estudiante 4, hombre, 15 años)

Cabe señalar que, frente a estas conductas de los docentes, los y las estudiantes reconocen no confrontarlas, pues señalan que los y las docentes cuentan con mayor apoyo por parte de las autoridades de la institución y, por ende, tienen mayor poder sobre ellos. Una estudiante comparte la siguiente reflexión:

Si me cae mal un profe ya no hay pa que discutir con él; no me hago el tiempo de discutir con él porque al final siempre va a terminar ganando el profe porque, por ejemplo, la directora obviamente va a defender al profe. Entonces como que no sacai na en discutir con el profe porque al final siempre ellos van a terminar ganando. (Estudiante 6, mujer, 15 años) 
En cuanto a las emociones vinculadas a la relación con pares en el contexto educativo, solo algunos estudiantes reportan emociones negativas de malestar. Estas se asocian con situaciones de bullying, problemas de convivencia, falta de interés por generar lazos de parte de sus compañeros y el no integrarlos en grupos de trabajo. Este último elemento es descrito como una consecuencia de ser nuevos en el curso y no por su pertenencia al programa PIE. Una estudiante ejemplifica esta idea señalando:

Aquí yo llegué hace rato ya igual, pero, por ejemplo, como ellas se conocen desde antes como que se dan más con ella, como su grupito. Entonces a veces como que me quedo sola así o me toca con las personas que no hablo tanto. Entonces yo siento que no voy a estar haciendo el trabajo y ya a lo mejor no hago el trabajo, y eso prefiero quedarme en la casa. (Estudiante 6, mujer, 15 años)

Las ideas anteriores evidencian que el compromiso emocional de los estudiantes se configura a partir de las relaciones que entablan con personas clave para su proceso educativo, destacándose emociones tanto positivas como negativas con respecto a la experiencia escolar, vinculadas a las acciones realizadas por docentes y pares.

\section{Compromiso cognitivo}

Vinculados al compromiso académico, los entrevistados mencionan variables personales que asocian a su rendimiento académico. El rendimiento es entendido como las notas que obtiene el estudiante en sus evaluaciones y el promedio de notas en general. Las variables descritas por la mayoría de los estudiantes asociados al rendimiento académico son el esfuerzo, la motivación y la atención. Señalan que el tener un alto nivel en cualquiera de estas variables se asociaría a un alto rendimiento. En contraste, tener un bajo nivel en dichas variables se asociaría a un rendimiento bajo.

El esfuerzo es comprendido por los y las participantes como el tiempo que dedican al estudio dentro y fuera del aula de clases, que se demuestra en el constante interés en clases, estudiar diariamente, resolver dudas con los/as docentes o pares, o prepararse con antelación para las evaluaciones.

Estudiante: Me pongo en mi pieza a estudiar o le pido ayuda a mis compañeras para que me expliquen lo que no entiendo...; eso es lo que hago. O le pido ayuda a las tías del PIE. Entrevistadora: ¿Y estudias siempre que tienes pruebas?

Estudiante: Sí, todos los días estudio, porque..., si no se me olvida; entonces estudio. (Estudiante 5, mujer, 14 años) 
Pese a lo anterior, la mayoría de los estudiantes entrevistados identifican que su esfuerzo por lo general es bajo, ya que mencionan estudiar solo cuando hay evaluaciones o bien cuando la materia es más desafiante. Además, expresan que no dedican suficientes horas extras de estudio y a la realización de tareas posterior a la jornada escolar, señalando el desinterés por la materia como la principal razón.

No me esfuerzo tanto; por ejemplo, si tengo prueba y (...) le dije a una amiga que íbamos a salir, prefiero salir ya y la prueba. Por ejemplo, si llego a la casa como a las 8 , y ahí yo me pongo a escribir lo que se trata la materia pa'l torpedo. (Estudiante 6, mujer, 15 años)

Por otra parte, la motivación es descrita por los y las estudiantes como las acciones que estimulan el estudio, tener interés por la clase, participar de actividades del establecimiento y asistir a clases. En el caso de los entrevistados, la motivación se expresa en un continuo de negativo (bajo) a positivo (alto), en donde la baja motivación se relaciona con escaso interés en las clases, el establecimiento, el estudio y la asistencia a clases. Por el contrario, una alta motivación se refleja en un elevado interés por estos mismos elementos.

Existen dos tipos de motivación que se destacan en las entrevistas realizadas, predominando la motivación extrínseca. Esta se refiere, de acuerdo a los estudiantes, a aquellos estímulos que surgen desde su entorno y los motivan a rendir en sus actividades académicas. Entre ellos mencionan el recibir reconocimiento académico de parte de sus docentes, familia y pares; y el uso de incentivos tales como notas y anotaciones positivas de parte de los y las docentes.

Entrevistadora: ¿Hacen cosas para que te sientas motivada con las actividades escolares? Entrevistado: Dicen: les voy a poner una anotación positiva. (Estudiante 10, mujer, 15 años)

Por otro lado, la motivación intrínseca es descrita por algunos estudiantes como aquella que surge desde ellos mismos, de sus características personales, intereses y proyectos futuros.

Entrevistadora: ¿Esa es tu motivación principal de venir al liceo y estudiar e intentar que te vaya bien?

Entrevistado: Sí, sacarme buenas notas para poder ser alguien en la vida. Que me vaya bien ahora para que me vaya bien después. (Estudiante 8 , mujer, 14 años) 
Un último elemento señalado por los estudiantes asociado a la participación en clases refiere a la atención. Esta es entendida como el atender a las actividades que se realizan dentro del aula. Los y las estudiantes señalan que la atención se ve influida por el agrado o desagrado que tengan por la materia y la relación que tengan con el docente, las que influyen en que presten atención a la clase. En contraste, si se dan las condiciones opuestas la atención es baja, tal como mencionan dos estudiantes:

Hay por ejemplo materias en las que nunca me ha ido bien; en Matemáticas y ahora en Química no, porque la profe me aburre; entonces como que tampoco me esfuerzo en prestar atención y me da sueño. (Estudiante 6, mujer, 15 años)

Porque me gusta poner atención solamente a las clases de la profe C o en Biología porque la profe de Biología como que a veces igual como que se sienta a hablar contigo y es más bacán que haya como, por ejemplo, confianza entre tú y los profes. (Estudiante 6, mujer, 15 años)

\section{Compromiso social}

Esta categoría incluye las interacciones que el estudiante establece con sus pares dentro del contexto escolar, a propósito de las actividades y contenidos académicos (Wang et al., 2019). Cabe señalar que los y las estudiantes destacan en mayor medida interacciones sociales con sus pares que no se vinculan directamente con las actividades académicas, pero que ocurren en el contexto escolar. Al respecto, todos los entrevistados señalan como motivación principal para asistir a clases el interactuar con sus pares. Al describir el tipo de interacciones, la mayor parte se refiere simplemente el ver a sus pares, mientras que algunos señalan que sus pares son importantes para apoyarse emocionalmente, refiriendo a contarse problemas personales.

En menor medida, los y las estudiantes dan cuenta de interacciones con sus compañeros y compañeras vinculadas a actividades académicas, señalando genéricamente que sienten apoyo de parte de ellos y, en algunas ocasiones, aclaran que sus compañeros son importantes para resolver dudas sobre contenidos o les piden materiales prestados para desarrollar actividades escolares.

A partir de lo anterior, es posible señalar que las interacciones con pares en el contexto escolar configuran un ambiente psicológico que puede ser comprendido como las sensaciones que emergen de las interacciones que ocurren el entorno educativo y el cómo se dan las interacciones sociales dentro de este. 


\section{Discusión}

El principal objetivo de esta investigación fue analizar la influencia de la relación docente-estudiante en el compromiso escolar de acuerdo con la percepción que tienen los estudiantes con NEE de primer año de enseñanza media de establecimientos municipales. El análisis evidencia la existencia de distintas condicionantes que impactan la relación de los estudiantes con NEE y sus docentes; a la vez, permite constatar que los docentes tienen un importante rol en distintas dimensiones del compromiso escolar descritas en la literatura (Fredericks et al., 2004; Moreira et al., 2015; Pérez-Salas et al., 2021; Ramos-Díaz et al., 2016; Wang et al., 2019).

La relación con los y las docentes es reconocida de forma positiva, de acuerdo con la percepción que los estudiantes tienen de ciertas características personales de los y las docentes, como la amabilidad, paciencia, confianza y buen humor. Inspirar confianza para compartir temas académicos y personales es identificado por los estudiantes como un elemento clave en la buena relación. Esto evidencia la importancia de la necesidad de vinculación de los estudiantes tal como lo plantean Connell y Wellborn (1991), quienes señalan que los/las estudiantes necesitan sentirse seguros, conectados y valorados por los demás, lo cual estaría siendo acogido por docentes cercanos y receptivos a sus necesidades.

Además, los estudiantes señalan que la buena relación se vincula con dos dimensiones del quehacer docente: sus prácticas y el rol que tienen en la atención de sus NEE. En cuanto a las prácticas, la claridad del docente al explicar los contenidos y responder asertivamente a sus dudas, así como el uso de recursos didácticos innovadores son reconocidos como elementos que motivan la atención y participación de los estudiantes. Asimismo, el sentir apoyo académico por parte de los/as docentes y, en especial, apoyo personalizado en el contexto del programa PIE, es percibido positivamente por los estudiantes. Estos elementos del quehacer docente evidencian la incidencia de la labor pedagógica en la satisfacción de las necesidades de vinculación y competencia (Connell \& Wellborn, 1991) de los y las estudiantes. Por una parte, las acciones docentes antes descritas contribuyen a la seguridad y sentimiento de sentirse valorado, en tanto los y las docentes son receptivos a sus necesidades e inquietudes académicas. Además, el percibir que cuentan con apoyo para enfrentar los desafíos académicos podría conceptualizarse como una estrategia que contribuye a la competencia de los estudiantes, en tanto estos señalan que el apoyo facilita su comprensión. 
Es importante señalar que el rol de apoyo de los docentes PIE se da en un contexto académico distinto al aula regular, el cual es reconocido por los estudiantes como un contexto que les hace sentir seguros para resolver sus inquietudes sin sentirse expuestos en el grupo más amplio. En este sentido, el contexto del programa PIE se configura como un elemento mediador de la relación de los estudiantes y los docentes, al ser tanto un espacio de trabajo académico como un espacio de satisfacción de necesidades de vinculación y competencia. Si bien una mayor estructuración del programa PIE podría implicar un menor desarrollo y ejercicio de la autonomía de los estudiantes debido al trabajo más estructurado y supervisado, el apoyo percibido hacia el aprendizaje en el contexto de PIE es concordante con los hallazgos de Fredericks et al. (2019); estos señalan que en estudiantes con bajo rendimiento académico una mayor autonomía en el trabajo escolar se asocia a un menor compromiso escolar, a diferencia de lo que ocurre en estudiantes con alto rendimiento. Si bien los autores no incorporan específicamente a estudiantes con NEE en su estudio, dichos resultados permiten comprender la necesidad de estructuración de los procesos de aprendizaje para estudiantes con bajo logro académico, como los estudiantes con NEE, dado que el bajo logro es uno de los criterios para evaluar la incorporación de estudiantes con NEE en los PIE.

Lo anterior se explica debido a que las necesidades de autonomía y competencia entrarían en conflicto en estudiantes con NEE, puesto que situaciones de mayor autonomía, como el trabajo en el aula regular, podrían llevar al estudiante a experimentar menor sentido de competencia, pudiendo preferir, entonces, un trabajo más estructurado y supervisado (menos autónomo), como el que ocurre en el PIE, con tal de sentirse más competente.

Lo anterior evidencia que la relación con los docentes, influenciada por acciones y características de ellos, es parte del contexto de aprendizaje que nutre la forma en la cual los estudiantes conceptualizan su sí mismo (Fredricks et al., 2019). Además, estos resultados evidencian la compleja relación existente entre las necesidades de los y las estudiantes y su compromiso escolar, y el rol central de la relación con sus docentes.

En el caso específico del compromiso, la percepción de los estudiantes sobre la relación con sus docentes se vincula con distintas dimensiones del compromiso, con especial énfasis en las dimensiones comportamental, emocional y cognitiva. Los estudiantes identifican que sus conductas positivas (tales como interés y participación en el trabajo académico) se vinculan con acciones de los docentes referidas a apoyar el trabajo académico y a hacerles sentir que sus necesidades e intereses son acogidos en el aula. 
Asimismo, en el caso de las reacciones emocionales positivas hacia la escuela, reportadas por los estudiantes, señalan percibir apoyo en dos ámbitos: académico y emocional. En el primero, señalan que sus docentes los apoyan en la realización de las actividades académicas, los tratan justamente y los integran al trabajo en el aula. En el ámbito emocional, los estudiantes identifican el apoyo de los docentes en temas personales como un elemento significativo para su valoración de la escuela. En cuanto a la dimensión cognitiva, los y las estudiantes mencionan al reconocimiento académico y los incentivos positivos (v. g. notas, anotaciones positivas) como elementos que se vinculan con una mejor disposición hacia las actividades académicas.

Los hallazgos anteriores coinciden con lo identificado en estudios anteriores (Engels et al., 2021; Pérez-Salas et al., 2021) sobre la relevancia de la relación docente-estudiante en el compromiso escolar de los estudiantes. En este estudio, las conductas y actitudes de los y las docentes dirigidas a apoyar el trabajo académico y el bienestar personal de los estudiantes son elementos percibidos positivamente por estos últimos. Esto constata que, de acuerdo con la percepción de los estudiantes, los docentes tienen actitudes positivas hacia la participación de los estudiantes con NEE en el aula. Asimismo, se evidencia que las acciones de los docentes se orientan mayormente a satisfacer las necesidades de vinculación y competencia de los y las estudiantes, pero no así su autonomía. Es necesario indagar en futuras investigaciones si esto último se relaciona con la postura que tiene el o la docente sobre las capacidades académicas de los estudiantes con NEE, tal como lo señala de Verdier et al. (2017).

Cabe indicar que algunos estudiantes reportan conductas asociadas a desconexión (Skinner et al., 2009; Wang et al., 2019), tales como poco involucramiento en el aprendizaje, no participar en clases o incurrir en conductas disruptivas. Asociado a estas, los estudiantes mencionan una mala relación con sus docentes, quienes realizan conductas consideradas molestas, especialmente en el ámbito académico, tales como hacer clases monótonas, no tenerles paciencia y no responder preguntas. Este hallazgo enfatiza la importancia de la relación docente-estudiante, no solo en el compromiso escolar, sino además en la aparición de procesos desadaptativos en las distintas dimensiones del compromiso escolar cuando esta es percibida negativamente por los/as estudiantes. El rol protector de la relación docente-estudiante en la desconexión escolar es concordante con los hallazgos de Archambault et al. (2017), en estudiantes con comportamientos oposicionistas y en estudiantes con alto riesgo de deserción en Chile (Espinoza et al., 2019). 
Además, la existencia de conductas y actitudes asociadas a un compromiso escolar positivo en todos los estudiantes entrevistados, en paralelo a algunas conductas de desconexión por parte de alguno de ellos, evidencia, tal como plantean Wang et al. (2019), que estos elementos no son parte de un continuo, sino que configuran dos constructos diferentes y separados.

Los hallazgos de esta investigación permiten posicionar la relevancia de la relación docente-estudiante en el compromiso escolar, lo que tiene el potencial de incidir en la trayectoria educativa de estudiantes secundarios con NEE. Estos resultados son relevantes de considerar por distintos actores educativos vinculados tanto al diseño de políticas educativas, formación inicial de docentes y docentes en ejercicio, para orientar la toma de decisiones y prácticas que posicionen la importancia de los vínculos afectivos y pedagógicos para promover y potenciar la inclusión educativa. Igualmente, los resultados de esta investigación permiten proyectar la necesidad de continuar estudiando el fenómeno incluyendo la perspectiva de los docentes para ampliar la comprensión de la relación docente-estudiante y los desafíos de la docencia para fortalecer el compromiso escolar de estudiantes secundarios con NEE. Asimismo, futuros estudios podrían ampliar el tema de estudio a estudiantes con y sin NEE, de manera de ampliar la comprensión de la relación entre compromiso escolar y la relación docente-estudiante, considerando las similitudes y diferencias entre la relación pedagógica que puede darse en relación a las necesidades de apoyo académico de estos grupos de estudiantes.

\section{Referencias}

Alley, K. M. (2019). Fostering middle school students' autonomy to support motivation and engagement. Middle School Journal, 5o(3), 5-14. https://doi.org/g6ss

Archambault, I., Vandenbossche-Makombo, J. \& Fraser, S. L. (2017). Students' oppositional behaviors and engagement in school: The differential role of the student-teacher relationship. Journal of Child and Family Studies, 26(6), 1702-1712. https://doi.org/f99fz8

Ben-Yehuda, S., Leyser, Y., \& Last, U. (2009). Teacher educational beliefs and sociometric status of special educational needs (SEN) students in inclusive classrooms. International Journal of Inclusive Education, 14(1), 17-34. https://doi.org/btszv7

Blatchford, P., Bassett, P., Brown, P., \& Webster, R. (2009). The effect of support staff on pupil engagement and individual attention. British Educational Research Journal, 35(5), 661-686. https://doi.org/10.1080/01411920902878917 
Burns, E., Martin, A., \& Collie, R. (2018). Adaptability, personal best (PB) goals setting, and gains in students' academic outcomes: A longitudinal examination from a social cognitive perspective. Contemporary Educational Psychology, 53, 57-72. https://doi.org/ 10.1016/j.cedpsych.2018.02.001

Canales, M. (2006). Metodologías de investigación social. LOM.

Carter, E., Sisco, L., Brown, L., Brickham, D., \& Al-Khabbaz, Z. (20o8). Peer interactions and academic engagement of youth with developmental disabilities in inclusive middle and high school classrooms. American Journal on Mental Retardation, 113(6), 479-494. https://doi.org/10.1352/2008.113:479-494

Christenson, S. L., Reschly, A. L., \& Wylie, C. (Eds.), (2012). Handbook of research on student engagement. Springer. https://doi.org/10.1007/978-1-4614-2018-7

Connell, J. P., \& Wellborn, J. G. (1991). Competence, autonomy, and relatedness: A motivational analysis of self-system processes. En M. Gunnar, \& L. A. Sroufe (Eds.), Minnesota symposium on child psychology (pp. 43-77). The University of Chicago.

Cortiella, C., \& Horowitz, S. H. (2014). The state of learning disabilities: Facts, trends and emerging issues. National Center for Learning Disabilities.

Creswell, J. W. (2011). Educational research: Planning, conducting, and evaluating quantitative. Prentice Hall.

de Verdier, K., Fernell, E., \& Ek, U. (2017). Challenges and successful pedagogical strategies: Experiences from six Swedish students with blindness and autism in different school settings. Journal of Autism and Developmental Disorders, 48(2), 520-532. https:// doi.org/10.1007/s10803-017-3360-5

Díaz-Bravo, L., Torruco-García, U., Martínez-Hernández, M., \& Varela-Ruiz, M. (2013). La entrevista, recurso flexible y dinámico. Investigación en Educación Médica, 2(7), 162-167. https://doi.org/10.1016/S2007-5057(13) 72706-6

Douglas, G., Travers, J., McLinden, M., Robertson, C., Smith, E., Macnab, N., Powers, S., Guldberg, K., McGough, A., O'Donnell, M., \& Lacey, P. (2012). Measuring educational engagement, progress and outcomes for children with special educational needs: A review. National Council for Special Education. https://bit.ly/3p9L307

Engels, M., Spilt, J., Denies, K., \& Verschueren, K. (2021). The role of affective teacherstudent relationships in adolescents' school engagement and achievement trajectories. Learning and Instruction, 75. https://doi.org/gjqmsg

Erentaitè, R., Vosylis, R., Gabrialavičiūtè, I. \& Raižienè, S. (2018). How does school experience relate to adolescent identity formation over time? Cross-lagged associations 
between school engagement, school burnout and identity processing styles. Journal of Youth and Adolescence, 47(4), 760-774. https://doi.org/10.1007/s10964-017-0806-1 Espinoza, O., González, E., Castillo, D., \& Neut, S. (2019). Condicionantes de la retención estudiantil en escuelas de segunda oportunidad en Chile. Revista Latinoamericana de Ciencias Sociales Niñez y Juventud, 17(2), 1-27. https://doi.org/10.1160o/1692715x.17201

Ewe, L. (2019). ADHD symptoms and the teacher-student relationship: A systematic literature review. Emotional and Behavioural Difficulties, 24(2), 136-155. https://doi.org/gh8hfg Flick, U. (2004). Introducción a la investigación cualitativa. Morata.

Forlin, C., \& Chambers, D. (2011). Teacher preparation for inclusive education: Increasing knowledge but raising concerns. Asia-Pacific Journal of Teacher Education, 39(1), 17-32. https://doi.org/10.1080/1359866x.2010.540850

Fredricks, J. (2015). Academic engagement. En J. Wright (Ed.), The International encyclopedia of the social \& behavioral sciences (pp. 31-36). Elsevier. https://doi.org/g6sw

Fredricks, J., Blumenfeld, P., \& Paris, A. (2004). School engagement: Potential of the concept, state of the evidence. Review of Educational Research, 74(1), 59-109. https:// doi.org/10.3102/0034654307400105

Fredricks, J., Parr, A. K., Amemiya, J. L., Wang, M. T., \& Brauer, S. (2019). What matters for urban adolescents' engagement and disengagement in school: A mixed-methods study. Journal of Adolescent Research, 34(5), 491-527. https://doi.org/gfv79j

Glaser, B., \& Strauss, A. (1967). The discovery of grounded theory: Strategies for qualitative Research. Aldine Publishing Company.

Hamre, B. K., \& Pianta, R. C. (2001). Early teacher-child relationships and the trajectory of children's school outcomes through eighth grade. Child Development, 72, 625-638. https://doi.org/10.1111/1467-8624.00301

Hernández-Sampieri, R., \& Mendoza, C. (2018). Metodología de la investigación: las rutas cuantitativa, cualitativa y mixta. McGraw-Hill.

Kiefer, S. M., Alley, K. M., \& Ellerbrock, C. R. (2015). Teacher and peer support for young adolescents' motivation, engagement, and school belonging. RMLE Online, 38(8), 1-18. https://doi.org/10.1080/19404476.2015.11641184

Li, Y., \& Learner, R. M. (2011). Trajectories of school engagement during adolescence: Implications for grades, depression, delinquency, and substance use. Developmental Psychology, ${ }_{47}$ (1), 233-247. https://doi.org/10.1037/a0021307

Maxwell, J. A. (2019). Diseño de investigación cualitativa. Gedisa. 
Moreira, P., Bilimória, H., Pedrosa, C., Pires, M., Cepa, M., \& Mestre, M. (2015). Engagement with school students with special educational needs. International Journal of Psychology and Psychological Therapy, 15(3), 361-375.

Moreira, P., Dias, P., Vaz, F. M., \& Vaz, J. M. (2013). Predictors of academic performance and school engagement-Integrating persistence, motivation and study skills perspectives using person-centered and variable-centered approaches. Learning and Individual Differences, 24, 117-125. https://doi.org/10.1016/j.lindif.2012.10.016

Nakkura, M. (2020). Identity and possibility: Adolescent development and the potential of schools. En M. Sadowski (Ed.), Adolescents at school: Perspectives on youth, identity, and education (pp.11-22). Harvard Education Press.

O'Donnell, K., \& Reschly, A. (2020). Student engagement and learning: Attention, behavioral, and emotional difficulties in School. En A. J. Martin, R. A. Sperling, \& K. J. Newton (Eds.), Handbook of educational psychology and students with special needs (pp.557-583). Routledge.

Pérez-Salas, C. P., Parra, V., Sáez-Delgado, F., \& Olivares, H. (2021). Influence of teacherstudent relationships and special educational needs on student engagement and disengagement: A correlational study. Frontiers in Psychology, 12. https://doi.org/g6sx Quin, D. (2017). Longitudinal and contextual associations between teacher-student relationships and student engagement: A systematic review. Review of Educational Research, 87(2), 345-387. https://doi.org/10.3102/0034654316669434

Ramos-Díaz, E., Rodríguez-Fernández, A., Fernández-Zabala, A., Revuelta, L. \& Zuazagoitia, A. (2016). Apoyo social percibido, autoconcepto e implicación escolar de estudiantes adolescentes. Revista de Psicodidáctica, 21(2), 339-356. https://doi.org/g6s2

Reeve, J., Jang, H., Carrell, D., Jeon, S., \& Barch, J. (2004). Enhancing students' engagement by increasing teachers' autonomy support. Motivation and Emotion, 28(2), 147-169. https://doi.org/10.1023/b:moem.0000032312.95499.6f

Rimm-Kaufman, S. E., Baroody, A. E., Larsen, R. A. A., Curby, T. W., \& Abry, T. (2016). To what extent do teacher-student interaction quality and student gender contribute to fifth graders' engagement in mathematics learning? Journal of Educational Psychology, 107, 170-185. https://doi.org/10.1037/aoo37252

Rogers, M., Bélanger-Lejars, V., Toste, J. R., \& Heath, N. L. (2015). Mismatched: ADHD symptomatology and the teacher-student relationship. Emotional and Behavioural Difficulties, 20(4), 333-348. https://doi.org/10.1080/13632752.2014.972039 
Roorda, D., Koomen, H., Spilt, J., \& Oort, F. (2011). The influence of affective teacherstudent relationships on students' school engagement and achievement. Review of Educational Research, 81(4), 493-529. https://doi.org/10.3102/0034654311421793

Ryan, R. M., \& Deci, E. L. (2017). Self-determination theory: Basic psychological needs in motivation, development, and wellness. Guilford Press.

Schindler, B. (2018). Student engagement in inclusive classrooms. Education Economics, 26(3), 266-284. https://doi.org/10.1080/09645292.2018.1426733

Skinner, E. A., Kindermann, T. A., Connell, J. P., \& Wellborn, J. G. (20og). Engagement as an organizational construct in the dynamics of motivational development. En K. Wentzel, \& A. Wigfield (Eds.), Handbook of motivation in school (pp. 223-245). Lawrence Erlbaum.

Skinner, E. A., \& Pitzer, J. R. (2012). Developmental dynamics of student engagement, coping, and everyday resilience. Springer.

Strauss, A., \& Corbin, J. (1994). Grounded theory methodology. En Denzin, N., \& Lincoln, Y. (Eds.), Handbook of qualitative research (pp. 273-285). Sage.

Wang, M., Brinkworth, M., \& Eccles, J. (2013). Moderating effects of teacher-student relationship in adolescent trajectories of emotional and behavioral adjustment. Developmental Psychology, 49(4), 690-705. https://doi.org/10.1037/a0027916

Wang, M., \& Eccles, J. (2012). Social support matters: Longitudinal effects of social support on three dimensions of school engagement from middle to high school. Child Development, 83(3), 877-895. https://doi.org/10.1111/j.1467-8624.2012.01745.x

Wang, M., \& Eccles, J. (2013). School context, achievement motivation, and academic engagement: A longitudinal study of school engagement using a multidimensional perspective. Learning and Instruction, 28, 12-23. https://doi.org/bs4p

Wang, M., \& Fredricks, J. A. (2014). The reciprocal links between school engagement, youth problem behaviors, and school dropout during adolescence. Child Development, 85(2), 722-737. https://doi.org/10.1111/cdev.12138

Wang, M., Fredricks, J., Ye, F., Hofkens, T., \& Linn, J. S. (2019). Conceptualization and Assessment of Adolescents' Engagement and Disengagement in School: A Multidimensional School Engagement Scale. European Journal of Psychological Assessment, 35(4), 592-606. https://doi.org/10.1027/1015-5759/aooo431

Wang, M., Willett, J., \& Eccles, J. (2011). The assessment of school engagement: Examining dimensionality and measurement invariance by gender and race/ethnicity. Journal of School Psychology, 49(4), 465-480. https://doi.org/10.1016/j.jsp.2011.04.001 